\title{
The Role of Serum Fibrinogen in Predicting Reinfection after DAIR (debridement, antibiotics, and implant retention) Treatment of Periprosthetic Joint Infections
}

\section{Dacheng Zhao}

Lanzhou University Second Hospital

He Jinwen

Lanzhou University Second Hospital

\section{Wang Xingwen}

Lanzhou University Second Hospital

\section{Zhao Xiaobing}

Lanzhou University Second Hospital

\section{Bin Geng}

Lanzhou University Second Hospital

\section{Yayi Xia (D 1016496744@qq.com )}

Lanzhou University Second Hospital

\section{Research article}

Keywords: periprosthetic joint infection, Debridement, implant retention, CRP, ESR, fibrinogen

Posted Date: February 2nd, 2021

DOI: https://doi.org/10.21203/rs.3.rs-154467/v1

License: (c) (i) This work is licensed under a Creative Commons Attribution 4.0 International License.

Read Full License 


\section{Abstract \\ Background}

Fibrinogen (FIB) has been used to differentiate periprosthetic joint infection (PJI) from aseptic loosening. The purpose of this study was to evaluate the diagnostic value of FIB in predicting postoperative reinfection in patients with debridement, antibiotics and implant retention (DAIR).

\section{Methods}

We retrospectively analyzed the patients who were admitted to DAIR from January 2013 to August 2019 for consideration of PJI readmission. Subgroups were divided into subgroups based on whether there was reinfection after DAIR treatment, and the diagnostic value of serum fibrinogen, erythrocyte sedimentation rate (ESR) and C-reactive protein (CRP) before DAIR treatment was analyzed by receiver operating Characteristic curve (ROC). To evaluate the diagnostic value of FIB in predicting postoperative reinfection in DAIR patients.

\section{Results}

FIB expression was different in acute PJI patients and chronic PJI patients treated with DAIR (4.03 VS 3.08; $\mathrm{P}<.05,4.28$ VS 3.68; $\mathrm{P}<.05)$. In patients with acute PJI treated with DAIR, the sensitivity and specificity of FIB were $81.82 \%$ and $83.33 \%$, respectively, significantly higher than CRP (sensitivity, $72.73 \%$; Specificity, 50\%; $P<.05$ ), while the specificity was higher than ESR (specificity, $41.67 \% ; P<.05$ ). In patients with chronic PJI treated with DAIR, the sensitivity and specificity of FIB were $80.00 \%$ and $66.66 \%$, respectively, significantly higher than CRP (sensitivity, 53.33\%; Specificity, 66.66\%; $P<.05$ ), ESR (sensitivity was $66.00 \%$; Specificity, $16.66 \%$; $<$.05).

\section{Conclusion}

FIB can predict reinfection after DAIR treatment for acute or chronic PJI. Considering the low success rate of DAIR treatment for chronic PJI, it should be chosen carefully.

\section{Introduction}

Periprosthetic joint infection (PJI) is a serious complication during total joint replacement and a major cause of failure after primary replacement [1]. According to the MSIS recommended criteria [2], acute infections are defined as those that occur within 90 days of the primary replacement, and chronic infections as those that occur more than 90 days after the primary replacement. Treatment options for PJI include DAIR, 1-stage revision, and 2-stage revision, joint fusion, and amputation [3-7]. The success rate of DAIR treatment after PJI diagnosis was $20-80 \%$, and it was related to the patients' obesity, 
comorbidities, operative time, perioperative period, microbial culture, and sensitivity to antibiotics [8-10]. For PJI patients, infection-free retention of implants after DAIR treatment is ideal. The international Consensus recommended DAIR for the treatment of acute PJI [11]. However, it has been reported that DAIR therapy for chronic PJI has achieved some success [12]. Infection after joint replacement is a challenge for orthopedic surgeons. The primary diagnosis depends on the patient's symptoms, imaging and laboratory examination, while the early evaluation of infection is usually performed by ESR, CRP and white blood cell (WBC) in the past. The above inflammatory markers have been widely used in the evaluation of infection after joint replacement [13-15]. However, the diagnosis of acute and chronic PJI is complex, CRP elevation in the acute phase can be affected by surgery, and MSIS does not recommend ESR for diagnosis in the acute phase [2]. Due to the formation of biofilms in chronic stage, it may be inaccurate to use ESR and CRP to evaluate infection alone [16]. Therefore, the assessment of acute and chronic PJI reinfection in DAIR after treatment may need new indicators, a prospective study of 2020 [17], CRP and fibrinogen as a diagnostic marker of infection, in terms of area under the curve (AUC), accuracy, sensitivity and specificity of the best, but combines several biomarkers to improve the accuracy of the diagnosis is not possible. A recent report [18] showed that plasma fibrinogen had the highest AUC (0.852), while D-dimer (a fibrin degradation product formed as an outcome of fibrin clot dissolution via plasmin) (0.657) and WBC (0.590) compared with the two classic markers, CRP and ESR (0.810 and 0.808 , respectively). Moreover, FIB is more valuable in terms of sensitivity, specificity, positive predictive value (PPV) and negative predictive value (NPV). In a previous retrospective study, we concluded that FIB can be used to identify aseptic loosening and chronic PJI, and that reinfection can be assessed after chronic PJI treatment with Spacer [19]. These studies suggest that FIB can be a good indicator of infection. However, whether fibrinogen, as an acute phase protein, can predict the incidence of reinfection after DAIR treatment in PJI patients has been relatively rarely reported. Therefore, the purpose of this study was to evaluate the diagnostic value of FIB in diagnosing reinfection in PJI patients after DAIR treatment.

\section{Materials And Methods}

This retrospective study was approved by the research Ethics Committee of our hospital. We conducted a retrospective study of 44 patients who were admitted to our hospital for DAIR treatment after PJI readmission due to total hip or total knee arthroplasty from January 2013 to August 2019. Inclusion criteria included: (1). Patients older than 18 years with PJI after primary total joint replacement and receiving DAIR therapy. (2). Acute PJI and chronic PJI were defined by the MSIS standard [2]. Acute was defined as infection occurring within 90 days after the primary replacement, while chronic was defined as infection occurring more than 90 days after the primary replacement. (3). Preoperative FIB, ESR and CRP values, as well as complete follow-up data. Exclusion criteria included: (1). A prior history of debridement or revision surgery on diseased joints. (2). Patients with a history of venous thromboembolism (VTE), antibiotic use two weeks before admission, anticoagulant use prior to admission, autoimmune disease, malignancy, liver disease, and data loss were excluded. The following cases are considered to be reinfected after DAIR treatment: (1). Any reoperation is required after surgery. (2). Long-term use of 
inhibitory antibiotics. (3). Recurrent infections are caused by strains of the same organism. (4). PJI related deaths. Finally, 44 patients were included, including acute PJI patients group ( 23 cases, group A) and chronic PJI patients group (21 cases, group B). They were divided into two subgroups, no infection and reinfection, according to whether reinfection occurred after DAIR treatment. Age, sex, body mass index and diseased joints were recorded. The demographic data of patients are shown in Table 1. The medical records of these patients, including gender, age, body mass index, comorbidities, diseased joints, diagnosis, surgical treatment, laboratory tests and follow-up records, were carefully recorded and reviewed. All patients were followed for at least 1 year.

Table 1

A cohort study of patients with acute PJI treated with DAIR.

\begin{tabular}{|lll|}
\hline Characteristic & $\begin{array}{l}\text { Reinfection } \\
\text { Yes }(\mathbf{n}=11) \text { No }(\mathbf{n = 1 2})\end{array}$ & P Value \\
\hline Age $(\mathrm{y})$ & $63.18 \pm 8.6263 .75 \pm 11.89$ & .898 \\
\hline Gender & $2(18.2 \%) 3(25.0 \%)$ & .912 \\
\hline Male & $9(81.8 \%) 9(75.0 \%)$ & .499 \\
\hline Female & $25.32 \pm 4.8826 .61 \pm 4.06$ & .296 \\
\hline BMl (kg/m2) & $6(54.5 \%) 10(83.3 \%)$ & \\
\hline Involved joint & $5(45.5 \%) 2(16.7 \%)$ & \\
\hline Knee & & \\
\hline Hip & & \\
\hline $\begin{array}{l}\text { PJI, periprosthetic joint infection; DAIR, debridement, antibiotics, and implant retention; BMl, body } \\
\text { mass index; SD, standard deviation. }\end{array}$ & \\
\hline All quantitative data are expressed as mean \pm SD. & \\
\hline
\end{tabular}

\section{Statistical analysis}

The AUC of FIB, CRP and ESR were calculated before [19], and the PASS software (version 15) was used to set the Power value as 0.9 to calculate the sample size required for this study, which was in line with the diagnostic efficacy. Continuous variables were expressed as mean and standard deviation, and baseline parameter data were analyzed by t-test. Frequency and composition ratio were determined by qualitative data, and chi-square test was used for classified data statistics. The rank sum test is used when the homogeneity of variance of continuous variables is not equal. The results after DAIR treatment were divided into reinfection and non-infection. The diagnostic value of each serological marker test before DAIR treatment was evaluated by the ROC curve. AUC value is interpreted as outstanding (0.90$1.00)$, good $(0.80-0.89)$, general $(0.70-0.79)$, difference $(0.60-0.69)$ or unqualified $(0.50-0.59)$. All 
statistical analyses were performed using IBM SPSS statistical software (Version 25). MedCalc statistical software (version 19) was used to analyze the ROC curve. Statistical significance was defined as $\mathrm{P}<0.05$.

\section{Results}

Sample characteristics

A total of 44 patients were included in this study. The success rate after DAIR treatment in acute PJI group (Group A) was $52.17 \%$ (12/23), and that after DAIR treatment in chronic PJI group (group B) was $28.57 \%(6 / 21)$. The demographic characteristics are shown in Table 1 and Table 2. There are no significant differences in age, sex, body mass index and diseased joints between group A and group B ( $P$ $>$.05; Table 1, Table 2).

Table 2

A cohort study of patients with chronic PJI treated with DAIR.

\begin{tabular}{|lll|}
\hline Characteristic & $\begin{array}{l}\text { Reinfection } \\
\text { Yes }(\mathbf{n}=15) \text { No }(\mathbf{n}=6)\end{array}$ & P Value \\
\hline Age $(\mathrm{y})$ & $64.27 \pm 9.1964 .50 \pm 3.51$ & .934 \\
\hline Gender & $9(18.2 \%) 350.0 \%)$ & .944 \\
\hline Male & $6(81.8 \%) 3(50.0 \%)$ & \\
\hline Female & $24.85 \pm 3.3325 .34 \pm 2.39$ & .749 \\
\hline BMl (kg/m2) & $9(54.5 \%) 4(66.7 \%)$ & .831 \\
\hline Involved joint & $6(45.5 \%) 2(33.3 \%)$ & \\
\hline Knee & & \\
\hline Hip & & \\
\hline $\begin{array}{l}\text { PJI, periprosthetic joint infection; DAIR, debridement, antibiotics, and implant retention; BMl, body } \\
\text { mass index; SD, standard deviation. }\end{array}$ & \\
\hline All quantitative data are expressed as mean \pm SD. & \\
\hline
\end{tabular}

Comparisons of biomarkers in each group

In the acute PJI group (group A) patients treated with DAIR, there was no significant difference in ESR and CRP (ESR 42.00 (38.00-65.00) VS 39.00 (20.25-49.50) mm/h) ( $>$ > .05; Table 3), (CRP 48.60 (27.5488.50) VS 61.44 (38.93-103.12) mg/L) ( $P>.05$; Table 3), FIB showed statistically significant differences among patients treated with DAIR in acute PJI group (Group A) (4.03 (3.45-4.61) VS 3.08 (2.92-3.32) $\mathrm{g} / \mathrm{L})(\mathrm{P}<.05 ;$ Table 3$)$. 
Table 3

Biomarker levels before DAIR therapy in patients with acute PJI.

\begin{tabular}{|llc|}
\hline Biomarkers & $\begin{array}{l}\text { Reinfection } \\
\text { Yes }(\mathbf{n}=11) \text { No( } \mathbf{n}=12)\end{array}$ & P Value \\
\hline ESR $(\mathrm{mm} / \mathrm{h})$ & $42.00(38.00-65.00) 39.00(20.25-49.50)$ & .288 \\
\hline CRP $(\mathrm{mg} / \mathrm{L})$ & $48.60(27.54-88.50) 61.44(38.93-103.12)$ & .651 \\
\hline FIB $(\mathrm{g} / \mathrm{L})$ & $4.03(3.45-4.61) 3.08(2.92-3.32)$ & $<.05$ \\
\hline $\begin{array}{l}\text { PJI, periprosthetic joint infection; DAIR, debridement, antibiotics, and implant retention; ESR, } \\
\text { erythrocyte sedimentation rate; CRP, C-reactive protein; FIB, fibrinogen. }\end{array}$ \\
\hline The data are expressed as median (interquartile range). \\
\hline
\end{tabular}

In the chronic PJI group (group B) treated with DAIR, there was no significant difference in ESR and CRP (ESR 34.00 (17.00-68.00) VS 35.50 (20.00-72.50) mm/h) (P > .05; Table 4), (CRP 23.26 (5.62-33.98) VS 13.06 (4.02-32.22) $\mathrm{mg} / \mathrm{L})(\mathrm{P}>.05$; Table 4), FIB showed statistically significant differences among patients treated with DAIR in the chronic PJI group (Group B) (FIB 4.28(3.98-5.12) VS 3.68 (3.20-4.05) $\mathrm{g} / \mathrm{L})(\mathrm{P}<.05 ;$ Table 4$)$.

Table 4

Biomarker levels before DAIR therapy in patients with chronic PJI.

\begin{tabular}{|llc|}
\hline Biomarkers & $\begin{array}{l}\text { Reinfection } \\
\text { Yes }(\mathbf{n}=15) \text { No( } \mathbf{n}=6)\end{array}$ & P Value \\
\hline ESR $(\mathrm{mm} / \mathrm{h})$ & $34.00(17.00-68.00) 35.50(20.00-72.50)$ & .733 \\
\hline CRP $(\mathrm{mg} / \mathrm{L})$ & $23.26(5.62-33.98) 13.06(4.02-32.22)$ & .622 \\
\hline FIB $(\mathrm{g} / \mathrm{L})$ & $4.28(3.98-5.12) 3.68(3.20-4.05)$ & $<.05$ \\
\hline $\begin{array}{l}\text { PJI, periprosthetic joint infection; DAIR, debridement, antibiotics, and implant retention; ESR, } \\
\text { erythrocyte sedimentation rate; CRP, C-reactive protein; FIB, fibrinogen. }\end{array}$ \\
\hline The data are expressed as median (interquartile range). \\
\hline
\end{tabular}

Analysis of ROC curve parameters of each group.

In acute PJI DAIR postoperative reinfection, ESR shows AUC is 0.63 , the best cutoff point 0.33 , sensitivity and specificity were $90.91 \%$ and $41.67 \%$ respectively, the positive predictive value and negative predictive value of $58.80 \%$ and $83.30 \%$ respectively, CRP shows AUC is 0.56 , the best cutoff point 0.23 , sensitivity and specificity were $72.73 \%$ and $50.00 \%$ respectively, the positive predictive value and negative predictive value were $57.10 \%$ and $66.70 \%$ respectively, FIB according to AUC is 0.75 , the best cutoff point of 0.65 , The sensitivity and specificity were $81.82 \%$ and $83.33 \%$, respectively. The positive predictive value and the negative predictive value were $81.80 \%$ and $83.30 \%$, respectively (Fig. 1 ; Table 5). 
Table 5

ROC curve parameters of acute and chronic PJI treated with DAIR for reinfection

\section{ROC Curve}

Parameters

Acute PJI ( $n=23)$, Reinfection ( $n=$

11)

Chronic PJI $(n=21)$, Reinfection $(n=$

15)

ESR

AUC

0.63

0.56

Optimal cutoff

$22 \mathrm{~mm} / \mathrm{h}$

$20 \mathrm{~mm} / \mathrm{h}$

Youden index

0.33

0.23

Sensitivity

$90.91 \%$

$41.67 \%$

$58.80 \%$

$83.30 \%$

$66.00 \%$

Specificity

PPV

NPV

0.56

$58.62 \mathrm{mg} / \mathrm{L}$

0.23

$72.73 \%$

$50.00 \%$

$57.10 \%$

$66.70 \%$

0.58

Optimal cutoff

Youden index

Sensitivity

Specificity

PPV

NPV

FIB

AUC

0.75

$3.32 \mathrm{~g} / \mathrm{L}$

0.65

$81.82 \%$

$83.33 \%$

$81.80 \%$

$83.30 \%$

NPV

Specificity

PPV

ROC, receiver operating characteristic; ESR, erythrocyte sedimentation rate; CRP, C-reactive protein; FIB, fibrinogen; AUC, area under the curve; PPV, positive predictive value; NPV, negative predictive value

\section{$16.50 \mathrm{mg} / \mathrm{L}$}

0.37

$53.33 \%$

$66.66 \%$

$80.00 \%$

$36.36 \%$
Optimal cutoff

$3.94 \mathrm{~g} / \mathrm{L}$

Youden index

0.63

Sensitivity

$80.00 \%$

$66.66 \%$

$85.71 \%$

$57.14 \%$ 
To chronic postoperative reinfection, ESR shows AUC is 0.56 , the best cutoff point 0.23 , sensitivity and specificity were $66.00 \%$ and $16.66 \%$ respectively, the positive predictive value and negative predictive value were $66.66 \%$ and $16.66 \%$ respectively, CRP shows AUC is 0.58 , the best cutoff point 0.37 , sensitivity and specificity were $53.33 \%$ and $66.66 \%$ respectively, the positive predictive value and negative predictive value were $80.00 \%$ and $36.36 \%$ respectively, FIB according to AUC is 0.81 , the best cutoff point of 0.63 ,The sensitivity and specificity were $80.00 \%$ and $66.66 \%$, respectively. The positive predictive value and the negative predictive value were $85.71 \%$ and $57.14 \%$, respectively (Fig. 2 ; Table 5).

\section{Discussion}

In the present study, our results suggest that (1) FIB is significantly associated with reinfection after DAIR treatment compared with traditional inflammatory indicators CRP and ESR. (2) Fibrinogen may be a promising indicator for predicting acute and chronic PJI after DAIR treatment. The literature review confirms that this study appears to be the first to evaluate the use of fibrinogen in predicting reinfection after treatment with DAIR for acute and chronic PJI.

PJI is a catastrophic complication after total joint replacement, which is the main reason for failure after primary replacement [1]. PJI can be classified into acute and chronic PJI according to MSIS criteria [2]. PJI treatment is a huge challenge for orthopedic surgeons. Treatment options for PJI include DAIR, primary or secondary revision arthroplasty, joint fusion, and amputation $[3,4,20]$. When acute PJI occurs after joint replacement, it may be difficult for orthopedic surgeons and patients to choose the treatment, because the results of preserving the prosthesis or undergoing revision surgery phase I or II are different, and improper treatment choices will lead to increased burden on patients. The international Consensus recommended DAIR for acute PJI [11]. If the implant can be retained without infection, the patient can avoid having to undergo the surgery again, avoiding the risk of complications. Our results showed that the success rate of DAIR treatment in acute PJI was $52.17 \%$, which was relatively close to the previously reported results [21]. CRP and ESR, as traditional inflammatory indicators, have been widely used to evaluate and predict post-replacement infection [15, 22, 23]. A study by Kuiper et al. [24] showed that when ESR was greater than $60 \mathrm{~mm} / \mathrm{h}$, the risk of DAIR treatment failure would increase. A multicentric retrospective study showed [9] that high CRP was associated with DAIR treatment failure. However, CRP is also elevated during the acute phase by the operation itself, and ESR is not recommended as a diagnostic indicator during the acute phase [2]. This requires a new indicator as a supplement to predict the outcome of DAIR treatment. FIB, as a coagulation test indicator, has recently been favored in the diagnosis of PJI. Recent evidence shows that fibrinogen can be used in the diagnosis of PJI and is a practical and economic marker [25]. Our previous studies have shown that FIB can be a good indicator for differentiating aseptic loosening and PJI [19]. Therefore, FIB was designed in this study to evaluate and predict the risk of reinfection in PJI patients treated with DAIR. Our results showed that compared with CRP and ESR, FIB predicted the maximum AUC of reinfection in the acute stage by 0.75 , and the sensitivity and specificity were different compared with CRP and ESR. The above results suggest that FIB appears to have some advantages in predicting acute PJI reinfection treated by DAIR. 
The treatment failure rate of DAIR for chronic PJI is high [26], However, DAIR can be used as a treatment for patients with immune dysfunction, comorbidities, and reluctance to undergo revision surgery phase I or II [27]. Studies have shown [28] that DAIR has been successfully applied to chronic PJI. Our results showed that the failure rate of DAIR in the treatment of chronic PJI was $71.43 \%$, and the AUC of FIB in the prediction of chronic reinfection was 0.81 , with sensitivity and specificity of $80.00 \%$ and $66.66 \%$, respectively, which seemed to indicate that the increase of FIB may indicate an increased risk of reinfection. However, a recent study [29] showed that the AUC of ESR and CRP in predicting the reinfection of chronic PJI after DAIR treatment was 0.48 and 0.27 respectively, and the sensitivity and specificity were $67 \%, 47 \%$ and $50 \%, 26 \%$, respectively. Their results also showed that CRP and ESR showed poor predictive efficacy of reinfection. However, this is related to the inconsistency of AUC, critical value, specificity and sensitivity of each indicator. In interpreting the above data and results, patient selection bias, sample size, study population, drug sensitivity test, treatment regimen, patient comorbidities, perioperative period, and PJI definition should also be taken into account.

There are some limitations to our study that should be taken into account in interpreting our findings. First of all, the study was a retrospective design, and it had some inherent biases. In addition, we chose serum FIB, and the results of plasma FIB may vary, and our sample size in each group is limited. Finally, there are many factors leading to the failure of DAIR treatment, such as the choice of surgical timing, bacterial culture results, drug sensitivity, comorbidities, etc. [30, 31], which need to be homogenized. Therefore, the design of a prospective, multicenter, large sample study is necessary to further confirm our findings.

\section{Conclusion}

FIB can predict reinfection after DAIR treatment for acute or chronic PJI. Considering the high failure rate of DAIR therapy in chronic PJI, it should be chosen carefully.

\section{Abbreviations}

CRP: C-reactive protein; ESR: Erythrocyte sedimentation rate; FIB: Fibrinogen; MSIS: Musculoskeletal Infection Society; PJI: Periprosthetic joint infection; DAIR: debridement, antibiotics and implant retention; WBC: white blood cell; ROC:

receiver operating Characteristic curve; AUC: area under the curve; PPV: positive predictive value ; NPV: negative predictive value; VTE: venous thromboembolism;

\section{Declarations}

\section{Funding}

This study was supported by The National Natural Science Foundation of China $(81874017,81960403$ and 82060405); Natrual Science Foundation of Gansu Province of China (20JR5RA320); Lanzhou 
Science and Technology Plan Program (2016-3-121, 2018-3-52); Cuiying Scientific and Technological Innovation Program of Lanzhou University Second Hospital (CY2017-QN12, CY2017-ZD02)囚The Fundamental Research Funds for the Central Universities (Izujbky-2020-kb17).

\section{Competing Interests}

The authors declare that there is no conflict of competing financial and non-financial interests.

\section{Availability of data and material}

Not applicable

\section{Code availability}

Not applicable

\section{Ethical Approval}

This retrospective study was approved by the research Ethics Committee of our hospital. Ethics approval number: 2019A-211.

\section{Consent to Participate}

All patients agreed to participate in the study.

\section{Consent to Publish}

All patients agreed to have their data published.

\section{Authors Contributions}

Xia Yayi and Geng Bin contributed to the study conception and design. Material preparation, data collection and analysis were performed by Zhao Dacheng $\triangle H e$ Jinwen and Wang Xingwen. The first draft of the manuscript was written by Zhao Dacheng and all authors commented on previous versions of the manuscript. All authors read and approved the final manuscript.

\section{References}

1. Parvizi J, Shohat N, Gehrke T. (2017) Prevention of periprosthetic joint infection: new guidelines. The bone \& joint journal 99-b (4 Supple B):3-10. doi:10.1302/0301-620x.99b4.Bjj-2016-1212.R1 .

2. Parvizi J, Gehrke T. Definition of periprosthetic joint infection. J Arthroplasty. 2014;29(7):1331. doi:10.1016/j.arth.2014.03.009.

3. Achermann Y, Stasch P, Preiss S, Lucke K, Vogt M. Characteristics and treatment outcomes of 69 cases with early prosthetic joint infections of the hip and knee. Infection. 2014;42(3):511-9. 
doi:10.1007/s15010-014-0584-6.

4. Di Benedetto P, Di Benedetto ED, Salviato D, Beltrame A, Gissoni R, Cainero V, Causero A. Acute periprosthetic knee infection: is there still a role for DAIR? Acta bio-medica. Atenei Parmensis. 2017;88(2s):84-91. doi:10.23750/abm.v88i2-S.6518.

5. Sherrell JC, Fehring TK, Odum S, Hansen E, Zmistowski B, Dennos A, Kalore N, Periprosthetic Infection C. The Chitranjan Ranawat Award: fate of two-stage reimplantation after failed irrigation and debridement for periprosthetic knee infection. Clin Orthop Relat Res. 2011;469(1):18-25. doi:10.1007/s11999-010-1434-1.

6. Tan TL, Goswami K, Fillingham YA, Shohat N, Rondon AJ, Parvizi J. Defining Treatment Success After 2-Stage Exchange Arthroplasty for Periprosthetic Joint Infection. J Arthroplasty. 2018;33(11):3541-6. doi:10.1016/j.arth.2018.06.015.

7. Kuo FC, Goswami K, Shohat N, Blevins K, Rondon AJ, Parvizi J. Two-Stage Exchange Arthroplasty Is a Favorable Treatment Option Upon Diagnosis of a Fungal Periprosthetic Joint Infection. J Arthroplasty. 2018;33(11):3555-60. doi:10.1016/j.arth.2018.07.024.

8. Löwik CAM, Parvizi J, Jutte PC, Zijlstra WP, Knobben BAS, Xu C, Goswami K, Belden KA, Sousa R, Carvalho A, Martínez-Pastor JC, Soriano A, Wouthuyzen-Bakker M. Debridement, Antibiotics, and Implant Retention Is a Viable Treatment Option for Early Periprosthetic Joint Infection Presenting More Than 4 Weeks After Index Arthroplasty. Clin Infect Dis. 2020;71(3):630-6. doi:10.1093/cid/ciz867.

9. Lora-Tamayo J, Murillo O, Iribarren JA, Soriano A, Sánchez-Somolinos M, Baraia-Etxaburu JM, Rico A, Palomino J, Rodríguez-Pardo D, Horcajada JP, Benito N, Bahamonde A, Granados A, del Toro MD, Cobo J, Riera M, Ramos A, Jover-Sáenz A, Ariza J. A large multicenter study of methicillin-susceptible and methicillin-resistant Staphylococcus aureus prosthetic joint infections managed with implant retention. Clin Infect Dis. 2013;56(2):182-94. doi:10.1093/cid/cis746.

10. Rodríguez-Pardo D, Pigrau C, Lora-Tamayo J, Soriano A, del Toro MD, Cobo J, Palomino J, Euba G, Riera M, Sánchez-Somolinos M, Benito N, Fernández-Sampedro M, Sorli L, Guio L, Iribarren JA, Baraia-Etxaburu JM, Ramos A, Bahamonde A, Flores-Sánchez X, Corona PS, Ariza J. Gram-negative prosthetic joint infection: outcome of a debridement, antibiotics and implant retention approach. A large multicentre study. Clin Microbiol Infect. 2014;20(11):0911-9. doi:10.1111/1469-0691.12649.

11. Argenson JN, Arndt M, Babis G, Battenberg A, Budhiparama N, Catani F, Chen F, de Beaubien B, Ebied A, Esposito S, Ferry C, Flores H, Giorgini A, Hansen E, Hernugrahanto KD, Hyonmin C, Kim TK, Koh IJ, Komnos G, Lausmann C, Loloi J, Lora-Tamayo J, Lumban-Gaol I, Mahyudin F, Mancheno-Losa M, Marculescu C, Marei S, Martin KE, Meshram P, Paprosky WG, Poultsides L, Saxena A, Schwechter E, Shah J, Shohat N, Sierra RJ, Soriano A, Stefansdottir A, Suleiman LI, Taylor A, Triantafyllopoulos GK, Utomo DN, Warren D, Whiteside L, Wouthuyzen-Bakker M, Yombi J, Zmistowski B. (2019) Hip and Knee Section, Treatment, Debridement and Retention of Implant: Proceedings of International Consensus on Orthopedic Infections. J Arthroplasty 34 (2S):S399-S419. doi:10.1016/j.arth.2018.09.025. 
12. Gramlich Y, Johnson T, Kemmerer M, Walter G, Hoffmann R, Klug A. Salvage procedure for chronic periprosthetic knee infection: the application of DAIR results in better remission rates and infectionfree survivorship when used with topical degradable calcium-based antibiotics. Knee surgery, sports traumatology, arthroscopy:. official journal of the ESSKA. 2020;28(9):2823-34. doi:10.1007/s00167019-05627-8.

13. Fujimoto $T$, Kaneko $T$, Sunakawa $T$, Ikegami H, Musha Y. Elevation of fibrin degradation product (FDP) values prevents the negative conversion of serum CRP values after total knee arthroplasty. J Orthop. 2018;15(4):940-4. doi:10.1016/j.jor.2018.08.005.

14. Stambough JB, Curtin BM, Odum SM, Cross MB, Martin JR, Fehring TK. Does Change in ESR and CRP Guide the Timing of Two-stage Arthroplasty Reimplantation? Clin Orthop Relat Res. 2019;477(2):364-71. doi:10.1097/01.blo.0000533618.31937.45.

15. Schiffner E, Latz D, Thelen S, Grassmann JP, Karbowski A, Windolf J, Schneppendahl J, Jungbluth P. Normal CRP and WBC values in total hip arthroplasty (THA) with signs of loosening. Do we need a joint aspiration? J Clin Orthop Trauma. 2019;10(3):566-70. doi:10.1016/j.jcot.2018.09.011.

16. Arnold WV, Shirtliff ME, Stoodley P. Bacterial biofilms and periprosthetic infections. Instr Course Lect. 2014;63:385-91.

17. Klim SM, Amerstorfer F, Glehr G, Hauer G, Smolle MA, Leitner L, Leithner A, Glehr M. Combined serum biomarker analysis shows no benefit in the diagnosis of periprosthetic joint infection. Int Orthop. 2020;44(12):2515-20. doi:10.1007/s00264-020-04731-6.

18. Li R, Shao HY, Hao LB, Yu BZ, Qu PF, Zhou YX, Chen JY. Plasma Fibrinogen Exhibits Better Performance Than Plasma D-Dimer in the Diagnosis of Periprosthetic Joint Infection: A Multicenter Retrospective Study. J Bone Joint Surg Am. 2019;101(7):613-9. doi:10.2106/JBJS.18.00624.

19. Bin G, Xinxin Y, Fan L, Shenghong W, Yayi X. Serum Fibrinogen Test Performs Well for the Diagnosis of Periprosthetic Joint Infection. J Arthroplasty. 2020;35(9):2607-12. doi:10.1016/j.arth.2020.04.081.

20. Qasim SN, Swann A, Ashford R. The DAIR (debridement, antibiotics and implant retention) procedure for infected total knee replacement - a literature review. Sicot j. 2017;3:2. doi:10.1051/sicotj/2016038.

21. Koyonos L, Zmistowski B, Della Valle CJ, Parvizi J. Infection control rate of irrigation and debridement for periprosthetic joint infection. Clin Orthop Relat Res. 2011;469(11):3043-8. doi:10.1007/s11999-011-1910-2.

22. Ishii Y, Noguchi H, Takeda M, Sato J, Takayama S, Toyabe S. Characteristics and significance of fever during 4 weeks after primary total knee arthroplasty. Arch Orthop Trauma Surg. 2014;134(5):707-12. doi:10.1007/s00402-014-1949-0.

23. Dupont C, Rodenbach J, Flachaire E. The value of C-reactive protein for postoperative monitoring of lower limb arthroplasty. Ann Readapt Med Phys. 2008;51(5):348-57. doi:10.1016/j.annrmp.2008.01.014. 
24. Kuiper JW, Vos SJ, Saouti R, Vergroesen DA, Graat HC, Debets-Ossenkopp YJ, Peters EJ, Nolte PA. Prosthetic joint-associated infections treated with DAIR (debridement, antibiotics, irrigation, and retention): analysis of risk factors and local antibiotic carriers in 91 patients. Acta Orthop. 2013;84(4):380-6. doi:10.3109/17453674.2013.823589.

25. Klim SM, Amerstorfer F, Gruber G, Bernhardt GA, Radl R, Leitner L, Leithner A, Glehr M. Fibrinogen - A Practical and Cost Efficient Biomarker for Detecting Periprosthetic Joint Infection. Sci Rep. 2018;8(1):8802. doi:10.1038/s41598-018-27198-3.

26. Maillet M, Pavese P, Bruley D, Seigneurin A, François $P$. Is prosthesis retention effective for chronic infections in hip arthroplasties? A systematic literature review. European journal of clinical microbiology infectious diseases: official publication of the European Society of Clinical Microbiology. 2015;34(8):1495-502. doi:10.1007/s10096-015-2388-8.

27. Ji B, Zhang X, Xu B, Ren J, Guo W, Mu W, Cao L. The fate of immunocompromised patients in the treatment of chronic periprosthetic joint infection: a single-centre experience. Int Orthop. 2018;42(3):487-98. doi:10.1007/s00264-018-3763-8.

28. Gramlich Y, Walter G, Klug A, Harbering J, Kemmerer M, Hoffmann R. Procedure for single-stage implant retention for chronic periprosthetic infection using topical degradable calcium-based antibiotics. Int Orthop. 2019;43(7):1559-66. doi:10.1007/s00264-018-4066-9.

29. Maier SP, Klemt C, Tirumala V, Oganesyan R, van den Kieboom J, Kwon YM. Elevated ESR/CRP Ratio Is Associated With Reinfection After Debridement, Antibiotics, and Implant Retention in Chronic Periprosthetic Joint Infections. J Arthroplasty. 2020;35(11):3254-60. doi:10.1016/j.arth.2020.06.007.

30. Tornero E, Martínez-Pastor JC, Bori G, García-Ramiro S, Morata L, Bosch J, Mensa J, Soriano A. Risk factors for failure in early prosthetic joint infection treated with debridement. Influence of etiology and antibiotic treatment. J Appl Biomater Funct Mater. 2014;12(3):129-34. doi:10.5301/jabfm.5000209.

31. Bedair HS, Katakam A, Bedeir YH, Yeroushalmi D, Schwarzkopf R. A decision analysis of treatment strategies for acute periprosthetic joint infection: Early irrigation and debridement versus delayed treatment based on organism. J Orthop. 2020;22:246-50. doi:10.1016/j.jor.2020.04.003.

\section{Figures}




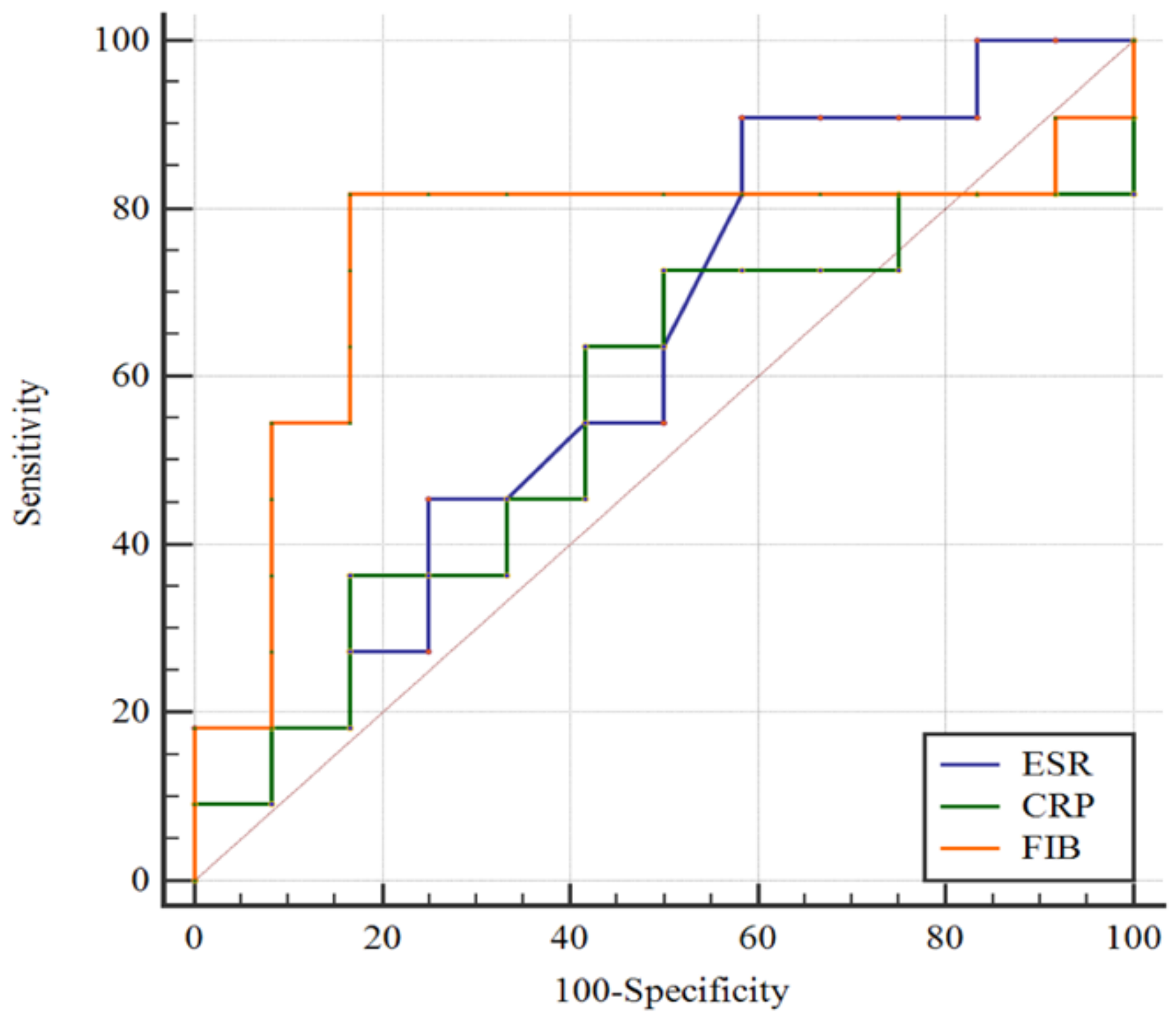

Figure 1

Receiver operating characteristic curve analysis for predicting reinfection after DAIR treatment for acute periprosthetic joint infection. DAIR, debridement, antibiotics, and implant retention; ESR, erythrocyte sedimentation rate; CRP, C-reactive protein; FIB, fibrinogen. 


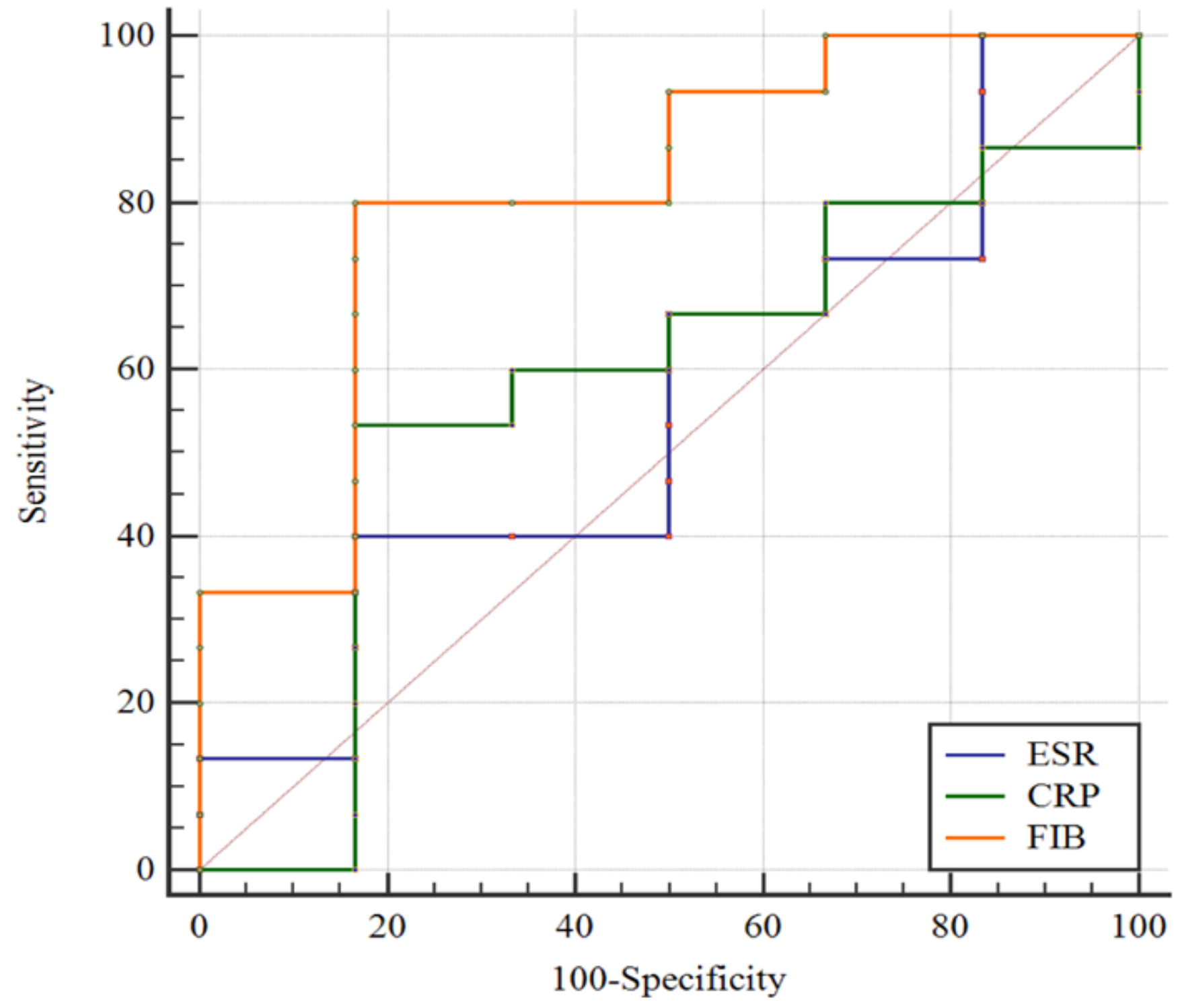

Figure 2

Receiver operating characteristic curve analysis for predicting reinfection after DAIR treatment for chronic periprosthetic joint infection. DAIR, debridement, antibiotics, and implant retention; ESR, erythrocyte sedimentation rate; CRP, C-reactive protein; FIB, fibrinogen. 